\title{
Sclerema neonatorum in a premature newborn
}

\section{Barbach Younes, Chaouche Mohammed, Dah Cherif Abdellah, Elloudi Sara, Baybay Hanane, Mernissi Fatima Zahra}

\author{
Departement of Dermatology, CHU Hassan II, Fès, Morocco
}

Corresponding author: Dr. Barbach Younes, E-mail: dr.younes2011@gmail.com

Sclerema of the newborn is classified among the lobular panniculitis, it is an extremely rare affection, most often lethal which occurs on a weak ground or sepsis [1]. The sclerema was announced for the first time at the beginning of the XVIIth century and the most authors have confused it with the scleroedema and cytosteatonecrosis of the newborn so the most diverse names have been given to these three conditions combined. Several theories have been proposed to explain his pathogenesis, which remains poorly understood [2]. The diagnosis of sclerema is clinical, it is manifested in newborns during their first week of life by a generalized cutaneous induration which gradually achieves, within a few days, a diffuse sclerous skin condition very paradoxically respecting the hands and feet, but may extend to compromising life-threatening dietary and respiratory functions. Sclerema treatment is based on newborn conditioning, antibiotic therapy, systemic corticosteroids, exsanguino-transfusion and

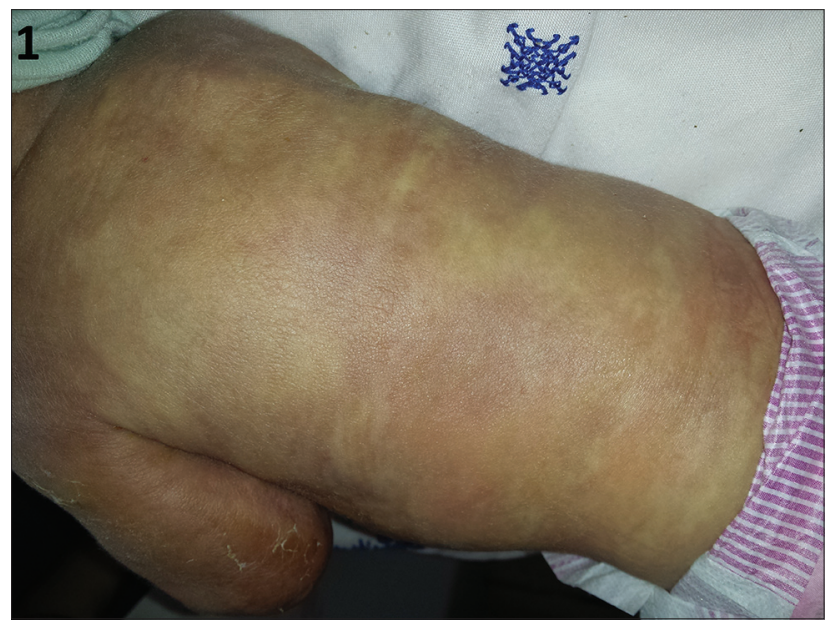

Figure 1: Diffuse back sclerosis. currently the advent of intravenous immunoglobulins. Despite these treatments, the prognosis of sclerema remains reserved with a high rate of mortality [3].

We report the case of a premature infant on Dl0 of life, hospitalized in neonatology for acute respiratory distress, which had since birth an induration of the diffuse skin. Dermatological examination showed the presence of a generalized sclerosis taking the whole body respecting the genitals as well as the palmoplantar region [Figs. 1-3], the diagnosis of newborn sclerema was retained after eliminating other diagnoses including neonatal cytosteotonecrosis and scleroderma, and then was put on cortico antibiotic combination.

\section{Consent}

The examination of the patient was conducted according to the Declaration of Helsinki principles.

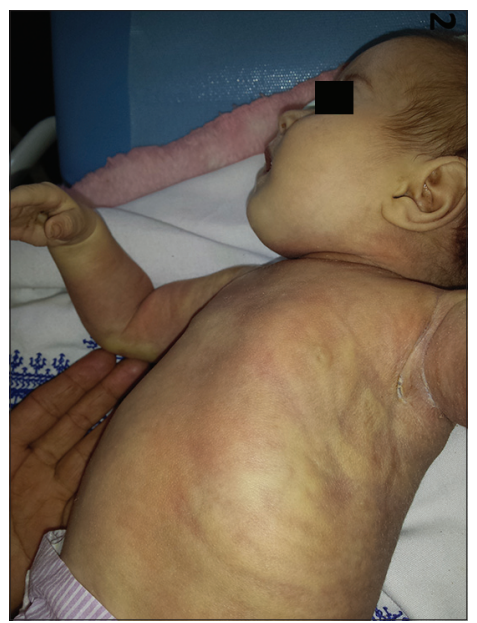

Figure 2: Generalized sclerosis.

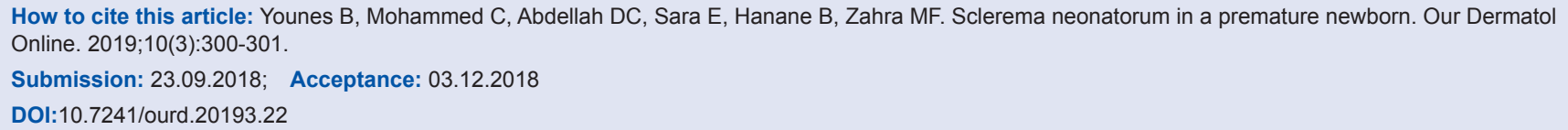


www.odermatol.com

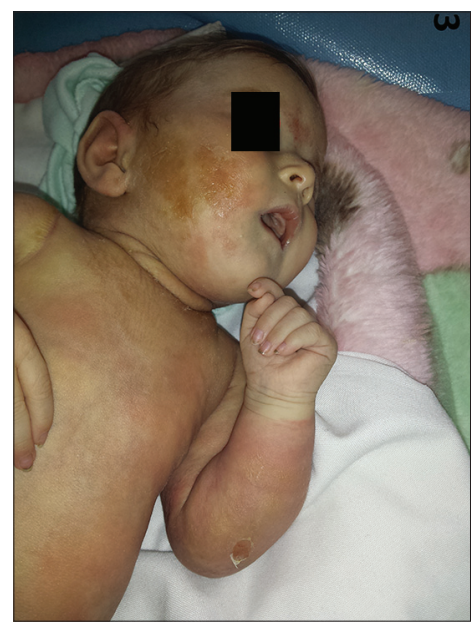

Figure 3: Sclerosis respecting palmar region.

\section{REFERENCES}

1. Park SH, Kim S-C. Sclerema neonatorum in a full-term infant showing favorable prognosis. Ann Dermatol. 2017;29:790-3.

2. Shrestha S, Chaudhary N, Koirala S, Gupta R. Sclerema neonatorum treated successfully with parenteral steroids: an experience from a resource poor country. Case Rep Pediatr. 2017;2017:1-2.

3. Zeb A, Darmstadt GL. Sclerema neonatorum: a review of nomenclature, clinical presentation, histological features, differential diagnoses and management. J Perinatol Off J Calif Perinat Assoc. 2008;28:453-60.

Copyright by Barbach Younes, et al. This is an open-access article distributed under the terms of the Creative Commons Attribution License, which permits unrestricted use, distribution, and reproduction in any

medium, provided the original author and source are credited.

Source of Support: Nil, Conflict of Interest: None declared. 